\title{
BMJ Open Pilot study to investigate the feasibility of the Home Falls and Accidents Screening Tool (HOME FAST) to identify older Malaysian people at risk of falls
}

Muhammad Hibatullah Romli, ${ }^{1,2}$ Lynette Mackenzie, ${ }^{1}$ Meryl Lovarini, ${ }^{1}$ Maw Pin $\operatorname{Tan}^{3}$

To cite: Romli MH, Mackenzie L, Lovarini M, et al. Pilot study to investigate the feasibility of the Home Falls and Accidents Screening Tool (HOME FAST) to identify older Malaysian people at risk of falls. BMJ Open 2016;6:e012048. doi:10.1136/bmjopen-2016012048

- Prepublication history for this paper is available online. To view these files please visit the journal online (http://dx.doi.org/10.1136/ bmjopen-2016-012048).

Received 24 March 2016 Revised 22 June 2016 Accepted 26 July 2016

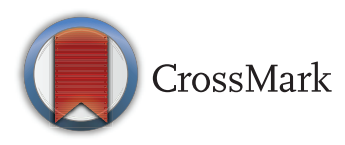

${ }^{1}$ Discipline of Occupational Therapy, University of Sydney, Lidcombe, New South Wales, Australia ${ }^{2}$ Department of Nursing and Rehabilitation, Faculty of Medicine and Health Sciences, Universiti Putra Malaysia, Serdang, Selangor, Malaysia

${ }^{3}$ Faculty of Medicine, University of Malaya, Malaysia

\section{Correspondence to} Muhammad Hibatullah Romli; mrom4634@uni.sydney.edu. $\mathrm{au}$

\section{ABSTRACT}

Objective: The relationship between home hazards and falls in older Malaysian people is not yet fully understood. No tools to evaluate the Malaysian home environment currently exist. Therefore, this study aimed to pilot the Home Falls and Accidents Screening Tool (HOME FAST) to identify hazards in Malaysian homes, to evaluate the feasibility of using the HOME FAST in the Malaysian Elders Longitudinal Research (MELoR) study and to gather preliminary data about the experience of falls among a small sample of Malaysian older people.

Design: A cross-sectional pilot study was conducted. Setting: An urban setting in Kuala Lumpur.

Participants: 26 older people aged 60 and over were recruited from the control group of a related research project in Malaysia, in addition to older people known to the researchers.

Primary outcome measure: The HOME FAST was applied with the baseline survey for the MELoR study via a face-to-face interview and observation of the home by research staff.

Results: The majority of the participants were female, of Malay or Chinese ethnicity and living with others in a double-storeyed house. Falls were reported in the previous year by $19 \%$ and $80 \%$ of falls occurred at home. Gender and fear of falling had the strongest associations with home hazards. Most hazards were detected in the bathroom area. A small number of errors were detected in the HOME FAST ratings by researchers. Conclusions: The HOME FAST is feasible as a research and clinical tool for the Malaysian context and is appropriate for use in the MELoR study. Home hazards were prevalent in the homes of older people and further research with the larger MELoR sample is needed to confirm the validity of using the HOME FAST in Malaysia. Training in the use of the HOME FAST is needed to ensure accurate use by researchers.

\section{INTRODUCTION}

Falls are a serious issue in Malaysia. What is known about falls in Malaysia is that $\sim 27 \%$ of

\section{Strengths and limitations of this study}

- This is the first kind of study in Malaysia investigating feasibility and transferability issues of a home hazards instrument in a local context.

- This pilot study successfully found specific cultural and demographic characteristics of older Malaysian people that may contribute to the presence of hazards in a home environment.

- There are a limited number of rigorous studies on home hazards and older people in Malaysia and this pilot study demonstrates the benefit of using an established standardised tool.

- The small sample size exposed the study findings to biases which require careful analysis and a larger scale study is required to confirm the findings.

- The study investigates the feasibility and transferability issues but not the psychometric properties of the tool to be used in the local context which require further investigation.

the rural community-dwelling Malaysian older people fall each year and half of them experience recurrent falls in the same year. ${ }^{1}$ In addition, the home is where older people frequently report injuries due to falls, second only to injuries from road accidents, ${ }^{2} 3$ and injuries at home for older people are most often related to falls. ${ }^{3}$

Three published Malaysian studies related to home hazards and falls have been conducted; ${ }^{1} 45$ however, findings on the association between home hazards and falls are inconclusive. Rizawati and Mas $\mathrm{Ayu}^{1}$ found no such association using the Safety House Checklist (SHC) and Home-Screen Scale (HSS) in their study, where both instruments were developed outside Malaysia. The HSS was developed for use by nurses, and the HSS and SHC are limited in the validity of 
their use to detect falls risk. Azhar and Md Yusof ${ }^{4}$ also found no association and did not use any standardised instrument to measure home hazards, and the authors developed their own measure. Their tool evaluated flooring conditions while other critical factors were left out, for instance, the use of grab bars in the toilet and bathroom. Significant associations were found in the Eshkoor $e t a \tilde{l}$ study, but the home hazards measurement was oversimplified into five environmental qualities, namely lighting, ventilation, noise, cleanliness and sanitation with simple evaluation criteria of low or high quality. It remains unclear from these studies how home environmental features contribute to falls risk.

Several limitations were observed related to the measurement instruments used in these studies: (1) limited demonstration of the psychometric properties of the tools, (2) the assessments were developed solely from a literature review or were self-developed, (3) some items in the assessments used were not related to falls hazards, (4) the assessments were not comprehensive or (5) the assessments lacked any functional items in relation to how an older person conducts functional tasks in the home environment. These studies also failed to accommodate the unique features of Malaysian culture which might influence the observation of hazards in home. For instance, Rizawati and Mas $\mathrm{Ayu}^{1}$ conducted their studies in a rural province in Malacca. Malaysian rural areas are likely to be culturally diverse, which will affect the physical and social environment compared with the kinds of home environments in countries where many home hazards tools have been developed.

The authors considered that the Home and Falls Accident Screening Tool (HOME FAST) was a suitable tool to thoroughly investigate home hazards and falls risk in Malaysia as the tool has been found to be associated with falls in an international study. ${ }^{6}$ The HOME FAST is psychometrically sound, ${ }^{7-11}$ is sensitive to change and detecting fall risk, ${ }^{6}$ and requires a short time to administer. ${ }^{79}{ }^{10}$ The tool has been developed to minimise cultural impact and allow universal application internationally. ${ }^{812}{ }^{13}$ However, a pilot study was needed to understand the feasibility and practicality of using the HOME FAST by Malaysian researchers, and in Malaysian homes. Therefore, the aims of this pilot study were to: (1) identify hazards in Malaysian homes using the HOME FAST, (2) evaluate the feasibility of using the HOME FAST for a Malaysian population and (3) gather preliminary data about the experience of falls among a small sample of Malaysian older people. This study would then provide useful information about the practicality of the HOME FAST and what may be required for its use in the larger population study.

\section{METHOD}

Design

This pilot study was conducted using a cross-sectional survey design as part of the initial baseline data collected for the Malaysian Elders Longitudinal Research (MELoR) study. Pilot studies are important to test the appropriateness of an instrument, to identify any potential logistical issues and to assess the feasibility of a fullscale study. ${ }^{14}$ For the use of the HOME FAST, this includes whether or not the instrument contains items of relevance to hazards in Malaysian homes, and if it can identify older people at higher risk of falls. Furthermore, we needed to test if there were cultural issues, such as the use of language, the interpretation of meanings and different everyday practices that may make the interpretation of HOME FAST items difficult for researchers to rate accurately. This required a pilot study to identify if the HOME FAST items are transferable to the Malaysian context and to gather initial information about the face validity of the tool. ${ }^{15} 16$

\section{Participants}

Research assistants working on the MELoR study were recruited to administer the HOME FAST. The research assistants came from a variety of academic backgrounds such as the built environment, biomedicine, education, economics, media art studies, engineering, psychology and occupational therapy. For the purpose of this pilot study, convenience sampling was used to recruit older people aged 60 years and over from the control group of a related research project in Malaysia, ${ }^{17}$ and additional older people were recruited who were known to the researchers. A sample size of between 24 and 36 has been identified as the optimal number for a pilot study, and this study sought to recruit a similar sample size. ${ }^{18}$ Participants were invited by telephone or in person to take part in this study. Consenting participants were provided with information explaining the objective of this study and a consent form to enable a home visit to be arranged.

\section{Data collection}

The baseline survey used for the MELoR study was applied to gather data on falls and demographic information. These data were used for analysis: demographic data, the HOME FAST items, fall history and fear of falls. Data were derived from structured interviews conducted with participants using the baseline survey. Falls in the previous year were self-reported using retrospective recall. Fear of falling was assessed by asking participants 'Are you afraid of falling?"19 and responses were recorded as 'yes' or 'no'.

A pair of researchers visited the participants at their homes to conduct the interviews. The interviews were conducted primarily in English as this was the language used in the original HOME FAST, and the baseline survey for the MELoR study. However explanations were provided for older people in their native language where required (usually Malay, Mandarin or Tamil). Only one of the researchers rated the HOME FAST on a single occasion with each of the older participants. 
Measurement tool for home hazards

An adapted version of the HOME FAST was used to measure home hazards for this study by reducing 25 items to 24. Items 18 and 19 from the original HOME FAST (rails on indoor steps and rails on outdoor steps) were merged into one general question about rails on indoor/outdoor steps in the home, in order to reduce the number of questions in the baseline MELoR survey. The adapted HOME FAST maintained the original format of the tool but did not include information about recommended interventions. The HOME FAST focuses on seven main areas of potential hazards: floors, furniture, lighting, bathroom, storage, stairways/steps and mobility. The HOME FAST scoring remained 'yes', 'no' and 'not applicable' (NA) for all the items. High HOME FAST scores corresponded to high numbers of hazards. Table 1 shows the HOME FAST items used in the MELoR study data collection.

The feasibility of research assistants administering the HOME FAST was evaluated by examining any inconsistencies or errors in data collection that could be observed, and through discussion. Issues such as ratings given by research assistants for the 'NA' HOME FAST items with Malaysian older people and challenges in accurately rating the HOME FAST by research assistants were explored through field notes completed during informal discussions in regular staff meetings.

\section{Analysis}

Statistical analyses were conducted using the SPSS V.20. Groups were compared using non-parametric methods. The Mann-Whitney U test, Kruskal-Wallis test and Spearman correlations were used to explore any associations with the HOME FAST score. Inconsistencies and errors on data keyed in the HOME FAST were observed for feasibility analysis.

\section{RESULTS}

\section{Participants}

Data from 26 older people were available for analysis. The mean age of participants was 68.69 years. Five participants $(19.2 \%)$ reported a fall in the previous year, and all of these were women. Twenty participants (77\%) admitted that they were having a fear of falling and the majority were women $(65 \%, \mathrm{n}=13)$. Table 2 summarises the characteristics of the participants including selfreported fall history and fear of falling responses.

\section{Hazards in the home}

Participants had a mean hazards score of 2.35 in their home (95\% CI 1.51 to 3.18) and the frequency of hazards detected was presented in table 3 as measured by the HOME FAST. Eight HOME FAST items (floor covering, chair access, toilet access, outside light, toilet

\begin{tabular}{|c|c|c|c|c|}
\hline & HOME FAST item & Yes & No & NA \\
\hline 1 & Are walkways free of cords and other clutter? & & & \\
\hline 2 & Are floor coverings in good condition? & & & \\
\hline 3 & Are floor surfaces non-slippery? & & & \\
\hline 4 & Are loose mats securely fixed to the floor? & & & \\
\hline 5 & Can the person get in and out of bed easily and safely? & & & \\
\hline 6 & Can the person get up from their lounge chair easily? & & & \\
\hline 7 & Are all the lights bright enough for the person to see clearly? & & & \\
\hline 8 & Can the person switch a light on easily from their bed? & & & \\
\hline 9 & Are the outside paths, steps and entrances well lit at night? & & & \\
\hline 10 & Is the person able to get on and off the toilet easily and safely? & & & \\
\hline 11 & Is the person able to get in and out of the bath easily and safely? & & & \\
\hline 12 & Is the person able to walk in and out of the shower recess easily and safely? & & & \\
\hline 13 & Is there an accessible/sturdy grab rail/s in the shower or beside the bath? & & & \\
\hline 14 & Are slip resistant mats/strips used in the bath/bathroom/shower recess? & & & \\
\hline 15 & Is the toilet in close proximity to the bedroom? & & & \\
\hline 16 & $\begin{array}{l}\text { Can the person easily reach items in the kitchen that are used regularly without climbing, bending or } \\
\text { upsetting his or her balance? }\end{array}$ & & & \\
\hline 17 & Can the person carry meals easily and safely from the kitchen to the dining area? & & & \\
\hline 18 & $\begin{array}{l}\text { Do the indoor steps/stairs have an accessible/sturdy grab rail extending along the full length of the } \\
\text { steps/stairs? }\end{array}$ & & & \\
\hline 19 & Can the person easily and safely go up and down the steps/stairs inside or outside the house? & & & \\
\hline 20 & Are the edges of the steps/stairs (inside and outside the house) easily identified? & & & \\
\hline 21 & Can the person use the entrance door/s safely and easily? & & & \\
\hline 22 & Are paths around the house in good repair, and free of clutter? & & & \\
\hline 23 & Is the person currently wearing well-fitting slippers or shoes? & & & \\
\hline 24 & If there are pets, can the person care for them without bending or being at risk of falling over? & & & \\
\hline
\end{tabular}


Table 2 Demographic data $(\mathrm{N}=26)$

\begin{tabular}{lll}
\hline Demographic & N (\%) & Mean $(95 \% \mathrm{Cl})$ \\
\hline Age & 68.69 \\
& $(66.08$ to 71.30$)$
\end{tabular}

\section{Gender}

Male

Female

Ethnicity

Malay

Chinese

Indian

Other

Religion

Islam

Christian

Buddhist

Hindu

Atheist

Marital status

Single/never married

Married

Widowed

Household

Alone

With others

Has maid

With spouse

Education

No schooling $2(7.7)$

Primary

Secondary

Certificate/skill

College/university

Type of house

Single-storey house

$11(42.3)$

$15(57.7)$

$11(42.3)$

$11(42.3)$

$3(11.5)$

1 (3.8)

10 (38.5)

$11(42.3)$

1 (3.8)

$3(11.5)$

$1(3.8)$

$2(7.7)$

$21(80.8)$

$3(11.5)$

5 (19.2)

$21(80.8)$

$6(28.5)$

$16(76.2)$

$1(3.8)$

$14(53.8)$

$1(3.8)$

$8(30.8)$

$6(23.1)$

$16(61.5)$

$4(15.4)$

Apartment-like or

condominium

Years living in the house

25.42

(19.65 to 31.19$)$

Has fallen in the past 12 months

Yes

$5(19.2)$

No

$21(80.8)$

Fall in home

Yes

No

$4(80)$

1 (20)

Afraid of falling

Yes

$20(76.9)$

No

$6(23.1)$

proximity, carrying meal, use of entrance, paths condition) were not identified as hazards.

\section{Administration of the HOME FAST in Malaysia}

Items 2, 13, 14, 17 and 23 (item 24 in the original HOME FAST) do not have the capacity to be scored as 'NA' in the original HOME FAST, but were incorrectly indicated as NA in this pilot study. For instance, item 2 (floor coverings in good condition) had three NA

Table 3 Hazards identified by the Home Falls and Accidents Screening Tool (HOME FAST) $(\mathrm{N}=26)$

\begin{tabular}{lr}
\hline Hazards & N (\%) \\
\hline No access to grab rails in bath & $11(42.3)$ \\
No slip resistant mats in the bath or shower & $10(38.5)$ \\
Improper footwear & $7(26.9)$ \\
Slippery floor surfaces & $6(23.1)$ \\
Hazardous pets & $5(19.2)$ \\
Unsecured loose mats & $4(15.4)$ \\
Poor lighting & $3(11.5)$ \\
Difficulty with shower mobility & $2(7.7)$ \\
Cluttered walkways & $2(7.7)$ \\
No access to bedside light & $2(7.7)$ \\
Difficult to identify stairways/steps edge & $2(7.7)$ \\
Inadequate stairs grab rail & $2(7.7)$ \\
Difficulty using stairs & $1(3.8)$ \\
Difficulty reaching items in kitchen & $1(3.8)$ \\
Difficulty with bath mobility & $1(3.8)$ \\
Difficulty with bed transfers & $1(3.8)$ \\
\hline
\end{tabular}

ratings-that is, no floor exists, item 13 (accessible/ sturdy grab rail/s in the shower or beside the bath) and item 14 (slip resistant mats/strips used in the bath/bathroom/shower recess) had four NA ratings-that is, no bath or shower exists, and item 23 (person currently wearing well-fitting slippers or shoes) had one NA rating - that is, the person has no feet. Item 5 (Can the person get in and out of bed easily and safely?) had one case of missing data, and item 12 (Is the person able to walk in and out of the shower recess easily and safely?) had two cases of missing data. Findings from discussions with the research assistants indicated that confusion appeared to arise about the differentiation between no and NA ratings for the HOME FAST. The purpose of a NA rating was that the feature was not present in the home and so could not be scored, whereas a no rating indicated the presence of a hazard -for example, the older person chose not to wear slippers or shoes. However, some raters interpreted this as NA because older people did not use the feature of the home regularly. Despite this, the raters agreed that all items on the HOME FAST were important to rate in Malaysian homes.

\section{Associations with the HOME FAST score}

A higher HOME FAST score was associated with being female and having a fear of falling. No other significant associations were found as reported in table 4 .

\section{DISCUSSION}

Findings related to home hazards in Malaysian homes identified by the HOME FAST

The results from this pilot study add to the understanding of the home environment and hazards in the homes of older people in Malaysia. This study shows a high frequency of hazards detected in parts of the home where 
Table 4 Associations with the Home Falls and Accidents Screening Tool (HOME FAST) score (N=26)

\begin{tabular}{|c|c|c|c|c|}
\hline & $\mathbf{N}$ & Median HOME FAST score & IQR & p Value \\
\hline \multicolumn{5}{|l|}{ Gender } \\
\hline Men & 11 & 1 & 2 & \multirow[t]{2}{*}{$0.016^{*} \dagger$} \\
\hline Women & 15 & 3 & 4 & \\
\hline \multicolumn{5}{|l|}{ Ethnicity } \\
\hline Malay & 11 & 2 & 2 & \multirow[t]{4}{*}{$0.453 \ddagger$} \\
\hline Chinese & 11 & 3 & 5 & \\
\hline Indian & 3 & 5 & 0 & \\
\hline Other & 1 & 1 & - & \\
\hline \multicolumn{5}{|l|}{ Living situation } \\
\hline Lives alone & 5 & 3 & 4 & \multirow[t]{2}{*}{$0.289 \dagger$} \\
\hline Lives with others & 21 & 2 & 4 & \\
\hline \multicolumn{5}{|l|}{ Has a maid } \\
\hline Yes & 6 & 1.5 & 2 & \multirow[t]{2}{*}{$0.556 \dagger$} \\
\hline No & 20 & 2 & 5 & \\
\hline \multicolumn{5}{|l|}{ Type of house } \\
\hline Any type of house & 22 & 2 & 4 & \multirow[t]{2}{*}{$0.971 \dagger$} \\
\hline Apartment & 4 & 2.5 & 3 & \\
\hline One floor & 10 & 1.5 & 4 & \multirow[t]{2}{*}{$0.503 \dagger$} \\
\hline Two floors & 16 & 2 & 4 & \\
\hline \multicolumn{5}{|l|}{ Falls history } \\
\hline Falls in the past 12-month & 5 & 5 & 4 & \multirow[t]{2}{*}{$0.155 \dagger$} \\
\hline No falls in the past 12-month & 21 & 2 & 3 & \\
\hline \multicolumn{5}{|l|}{ Afraid of falling } \\
\hline Yes & 20 & 2 & 4 & \multirow[t]{2}{*}{$0.033^{\star} \dagger$} \\
\hline No & 6 & 0.5 & 2 & \\
\hline
\end{tabular}

*Significant differences detected at $p<0.05$.

†Mann-Whitney U test.

$\ddagger$ Kruskal-Wallis test.

older people in Malaysia mostly fall, as reported in previous findings from Rizawati and Mas Ayu, ${ }^{1}$ which are the bathroom, stairs, living room, kitchen and bedroom. The types of hazards present in this study are similar to those reported by Byles et $a t^{20}$ in an Australian study such as absence of a grab rail in the shower or bath area and slippery floor. Hazards in the bathroom and toilet area warrant high priority for any Malaysian studies as Malaysians commonly practise self-care activities in a wet bathroom and wet toilet area, where water may be used by some older people to wash themselves by scooping water from a pail and rinsing in the area, or by using a hose.

However, the mean score on the HOME FAST in this study was lower than the mean scores generated from Australian study. The cut-off point on the HOME FAST for a high risk of falls associated with the number of home hazards has been stated as $\geq 8 .^{20}$ In the Australian study, $27 \%$ of the participants scored $\geq 8$. However, the highest score on the HOME FAST recorded in this study was six. It is possible that the low HOME FAST scores were due to the underestimation of hazards present based on the ratings from the research administrators, many of whom did not have health backgrounds. Mistakes in data collection and missing data might also have contributed to this result. Therefore, this study indicated a requirement for training for the nonoccupational therapy raters in scoring the HOME FAST.

\section{Feasibility of the HOME FAST for use in Malaysia}

The use of the HOME FAST was positively accepted by the researcher participants, although several challenges were noted. Only a small number of missing data were available in this study and were usually related to potentially sensitive issues requiring an older person to demonstrate an activity in a private area such as the bedroom and bathroom. Since the HOME FAST is conducted through interview and observation, there may be difficulties with gaining permission to observe the entire house, which may affect the ability to score the HOME FAST accurately. To be effectively measured, the HOME FAST items need to include an observation of how a person functions in their home environment. ${ }^{21}$ This requires the administrator to clearly discuss the aims and benefits of conducting the HOME FAST assessment to encourage an older person to be more willing to engage in the process.

The research assistants who administered the HOME FAST managed to detect several common hazards although they have little knowledge on hazards evaluation. This indicates that the HOME FAST is feasible to be used by people from a variety of backgrounds. However, two points need to be taken into account to improve the HOME FAST scoring process. First, different cultural understandings in interpreting the questions may have contributed to some confusion on how to 
score the HOME FAST items. ${ }^{15}{ }^{16}$ For example, in Malaysian culture, people usually need to take off their shoes before entering a house as wearing shoes inside a house is considered inappropriate and walking barefoot indoors is common. Thus, when administering the HOME FAST, the footwear item might be misinterpreted as 'yes' rather than 'no' (eg, wearing of socks in the house). Second, the varied backgrounds of the research staff may have limited their in-depth understanding of home hazards. Therefore, this may have affected the results as the scoring may not have been accurate based on differing interpretations of the HOME FAST items.

\section{Preliminary findings related to the home environment and self-reported falls}

The findings from this study are consistent with international studies. Although the reported prevalence of falls in this study of $19 \%$ is lower than the prevalence reported globally at $28-35 \%,{ }^{22}$ it is consistent with lower fall rates among Asian countries, ${ }^{123}$ and the fall rates are higher for older women compared with older men. ${ }^{24}$ Women may have had a higher number of hazards given that in Malaysia women usually have a role as a housewife and are more likely to be in the home environment for a longer time and are more likely to undertake functional tasks in the home ${ }^{25}$ they may also have greater role demands requiring their participation in domestic activities such as cooking and taking care of the family or looking after grandchildren, which could expose them to further hazards. ${ }^{25}{ }^{26}$ In addition, women often have more health conditions that contribute to frailty such as arthritis, low muscle strength and osteoporosis, which indirectly affects functioning. ${ }^{27}$

An unexpected finding from this study was the association between fear of falling and home hazards. This study used a simple measure of fear of falling compared with previous studies. ${ }^{28}$ Fear of falling may restrict the activities of an older person resulting in a decline in physical and mental performance, reduced quality of life and increased risk of falls. ${ }^{29}$ This may then make the older person less involved in household chores and activities which could increase the presence of home hazards and difficulties with transfers and so on. This finding is important as many older people tend to believe that falls occur due to their own behaviour, rather than because of environmental factors, which may cause a low take-up of home hazard management options. ${ }^{30} 31$ It is possible that managing environmental falls risks may reduce the fear of falls. ${ }^{32}$ This association can be explored in more detail in the larger MELoR study.

Although only gender and fear of falling were significantly associated with a higher number of hazards in this study, a larger study may reveal other factors associated with home hazards such as falls history, living situation and ethnicity. Even with a small sample, the study provides some initial direction about the characteristics of older people who are likely to have higher numbers of home hazards, and be at higher risk of falls, ${ }^{14} 33$ and who should therefore be targeted for more comprehensive fall prevention interventions.

\section{Implication for future study}

This is the first study investigating the feasibility of the home hazards instrument in the Malaysian context. Given the findings, the HOME FAST was considered a relevant tool for the Malaysian context, despite some feasibility issues to be overcome for it to be used effectively by the research assistants. The HOME FAST contains detailed descriptions to assist raters to effectively score any presenting hazards, and these must be maintained for use in Malaysia. In addition, the HOME FAST may need a forced answer (closed-ended) format to help the administrator not make any mistakes when scoring. Conducting the interviews in English did not appear to be a problem for the researchers in this pilot study. All the researcher participants were graduates from tertiary education, and it is compulsory in Malaysia for every person to pass an English examination before being accepted for study in tertiary education. ${ }^{34}$

The low numbers of hazards reported in this pilot study indicate that further investigation is needed to explore the validity and reliability of the HOME FAST in Malaysia. The difference in cultural understanding and educational backgrounds of the research staff might interfere with the ability to score the HOME FAST. The presence of irregularities in the HOME FAST data collected in this study warrants specific training to be conducted to maximise the reliability and consistency in the use of this assessment. ${ }^{1011} 14$ Therefore, this pilot study has demonstrated the need for further study of the psychometric properties of the HOME FAST in the Malaysian context.

The limitations of a small sample size are anticipated for pilot studies. Convenience sampling may have added some underlying bias to the results. For instance, the participants in this study might be healthier than the general population, and that may be why the hazard scoring is somewhat lower. The cross-sectional design of this study provides unclear study associations, and a larger longitudinal study such as the MELoR study will allow these associations to be tested further.

\section{CONCLUSION}

This study provides a good foundation to support the use of the HOME FAST in a larger study. The HOME FAST was practical and transferable to the Malaysian context, and with further training for the study researchers, the HOME FAST can be accurately applied and interpreted following the recommendations from this pilot study. As the MELoR project progresses, it is hoped that we can develop a better understanding about the role of home environment and the interaction of older people with their home environments and the risk of falls. 
Acknowledgements The authors thank the research assistants of the MELoR project who dedicated their time and effort in collecting the data required. They also thank the older people who volunteered to participate in this study.

Funding This study was funded by the Ministry of Higher Education, Malaysia and the University of Malaya (UM.C/625/1/HIR/MOHE/ASH/02).

Competing interests None declared.

Patient consent Obtained.

Ethics approval The study received ethics approval from the Ethical Committee, High Impact Research, University of Malaya (MEC Ref No: 943.6).

Provenance and Peer Review Not commissioned, externally peer reviewed.

Data sharing statement No additional data are available.

Open Access This is an Open Access article distributed in accordance with the Creative Commons Attribution Non Commercial (CC BY-NC 4.0) license, which permits others to distribute, remix, adapt, build upon this work noncommercially, and license their derivative works on different terms, provided the original work is properly cited and the use is non-commercial. See: http:// creativecommons.org/licenses/by-nc/4.0/

\section{REFERENCES}

1. Rizawati M, Mas Ayu S. Home environment and fall at home among the elderly in Masjid Tanah Province. JUMMEC: $J$ Health Transl Med 2008;11:72-82.

2. Hasni $\mathrm{H}$, Junainah $\mathrm{S}$, Jamaliah J. Epidemiology of home injury in Malaysia. J Kesihatan Masyarakat 2003;9:1-7.

3. Lim KH, Jasvindar K, Normal I, et al. Risk factors of home injury among elderly people in Malaysia. Asian J Gerontol Geriatr 2014;9:16-20.

4. Azhar AH, Md Yusof S. Fall risk factors among Malaysian older adults. J Phys Act Sports Exerc 2013;1:31-7.

5. Eshkoor SA, Hamid TA, Hassan Nudin SS, et al. The effects of sleep quality, physical activity, and environmental quality on the risk of falls in dementia. Am J Alzheimers Dis Other Demen 2013;28:403-7.

6. Mackenzie L, Byles J, D'Este C. Longitudinal study of the Home Falls and Accidents Screening Tool in identifying older people at increased risk of falls. Australas J Ageing 2009;28:64-9.

7. Mackenzie L, Byles J, Higginbotham N. Designing the Home Falls and Accidents Screening Tool (HOME FAST): selecting the items. Br J Occup Ther 2000;63:260-9.

8. Mackenzie L, Byles J, Higginbotham N. Professional perceptions about home safety: cross-national validation of the Home Falls and Accidents Screening Tool (HOME FAST). J Allied Health 2002;31:22-8.

9. Mackenzie L, Byles J, Higginbotham N. Reliability of the Home Falls and Accidents Screening Tool (HOME FAST) for identifying older people at increased risk of falls. Disabil Rehabil 2002;24:266-74

10. Vu TV, Mackenzie L. The inter-rater and test-retest reliability of the Home Falls and Accidents Screening Tool. Aust Occup Ther J 2012;59:235-42.

11. Hassani Mehraban A, Mackenzie LA, Byles JE. A self-report home environment screening tool identified older women at risk of falls. $J$ Clin Epidemiol 2011;64:191-9.

12. Maghfouri B, Mehraban AH, Taghizade G, et al. Internal consistency of reliability assessment of the Persian version of the 'Home Falls and Accident Screening Tool'. Iran Rehabil J 2013;11:46-50.

13. Guo QY, Guo MJ, Zhang L, et al. 居家跌倒风险篮查量表在中国社区 老年人中的应用 [Home fall risk screening scale application in
Chinese elderly in the community]. Chinese $J$ Nurs 2015;50:1128-32.

14. van Teijlingen E, Hundley V. The importance of pilot studies. Nurs Stand 2002;16:33-6.

15. Awaad T. Culture, cultural competency and occupational therapy: a review of the literature. Br J Occup Ther 2003;66:356-62.

16. Simkhada $P$, Bhatta $P$, van Teijlingen ER. Importance of piloting a questionnaire on sexual health research. Wilderness Environ Med 2006;17:295-6.

17. Tan PJ, Khoo EM, Chinna K, et al. An individually-tailored multifactorial intervention program for older fallers in a middle-income developing country: Malaysian Falls Assessment and Intervention Trial (MyFAIT). BMC Geriatr 2014;14:78-94.

18. Johanson GA, Brooks GP. Initial scale development: sample size for pilot studies. Educ Psychol Meas 2010;70:394-400.

19. Cumming RG, Salkeld G, Thomas M, et al. Prospective study of the impact of fear of falling on activities of daily living, SF-36 scores, and nursing home admission. J Gerontol A Biol Sci Med Sci 2000;55: M299-305.

20. Byles JE, Mackenzie L, Redman S, et al. Supporting housing and neighbourhoods for healthy ageing: findings from the Housing and Independent Living Study (HAIL). Australas J Ageing 2014:33:29-35.

21. Iwarsson S, Horstmann V, Carlsson G, et al. Person-environment fit predicts falls in older adults better than the consideration of environmental hazards only. Clin Rehabil 2009;23:558-67.

22. World Health Organisation. WHO Global Report on Falls Prevention in Older Age. France: World Health Organisation, 2007:1-47.

23. Kim EJ, Arai $\mathrm{H}$, Chan $\mathrm{P}$, et al. Strategies on fall prevention for older people living in the community: a report from a round-table meeting in IAGG 2013. J Clin Gerontol Geriatr 2015;6:39-44.

24. Gillespie LD, Robertson MC, Gillespie WJ, et al. Interventions for preventing falls in older people living in the community. Cochrane Database Syst Rev 2012;9:CD007146

25. Selvaratnam DP, Tin PB. Lifestyle of the elderly in rural and urban Malaysia. Ann N Y Acad Sci 2007;1114:317-25.

26. Mohamed A, Rostam K. The Malay elderly at the fringe of metropolitan region, Selangor: experience and perception of self. Geografia: Malaysian. J Soc Space 2012;8:1-14.

27. Gitlin LN, Mann W, Tomit M, et al. Factors associated with home environmental problems among community-living older people. Disabil Rehabil 2001;23:777-87.

28. Mahmood H, Talib SS, Embong AM, et al. The fear of falling among older adults: zooming in homes in Malacca, Malaysia. Paper Presented in 5th International Online Medical Conference; Vol 1, 2012:174.

29. Scheffer AC, Schuurmans MJ, van Dijk N, et al. Fear of falling: measurement strategy, prevalence, risk factors and consequences among older persons. Age Ageing 2008;37:19-24.

30. Petersson I, Lilja M, Borell L. To feel safe in everyday life at homea study of older adults after home modifications. Ageing Soc 2012;32:791-811

31. Lee F, Mackenzie L, James C. Perceptions of older people living in the community about their fear of falling. Disabil Rehabil 2008;30:1803-11.

32. Munski JL. The effect of an environment modification on fear of falling in institutionalized older persons [MSc dissertation]. Grand Valley State University, 1998.

33. Clemson L, Mackenzie L, Ballinger C, et al. Environmental interventions to prevent falls in community-dwelling older people: a meta-analysis of randomized trials. J Aging Health 2008;20: 954-71.

34. Hanapiah MF. English language and the language of development: a Malaysian perspective. J Kemanusiaan 2004;3:107-20. 